\title{
Cutaneous Adverse Drug Reaction: A Review of a Four-year Experience in a Tertiary Referral Hospital in Malaysia
}

\author{
Rajalingam Ramalingam ${ }^{1 *}$ \\ ${ }^{1}$ Department of Dermatology, Hospital Tengku Ampuan Afzan, Kuantan, Pahang, Malaysia
}

Corresponding Author: Rajalingam Ramalingam, Department of Dermatology, Hospital Tengku Ampuan Afzan, Kuantan, Pahang, Malaysia. E-mail: raj.blueheart@gmail.com

Received February 16, 2017; Accepted April 15, 2017; Online Published May 29, 2017

Introduction: There are many types of cutaneous adverse drug reactions (CADRs), from transient erythema to severe lifethreatening conditions such as Stevens-Johnson syndrome (SIS) and toxic epidermal necrolysis (TEN), with significant morbidity and mortality. Awareness of the local epidemiology of CADR may play a vital role in future clinical management protocols.

Methods: A retrospective review of all patients referred to the Department of Dermatology of Hospital Tengku Ampuan Afzan, Kuantan, Pahang, Malaysia, with confirmed CADR from 2013 to 2016 was carried out to determine the epidemiology of CADR in the local population.

Results: A total of 62 reactions involving 59 patients were seen among 7,353 new patients, yielding an incident rate of $0.8 \%$ (yearly CADR rate range: $0.16-1.89 \%$ ), with the highest rate seen among indigenous peoples $(2.53 \%)$. SJS (15 cases) was the most frequent CADR, followed by maculopapular eruption (13) and TEN (6) among others. Severe CADRs (SJS, TEN, drug-related eosinophilia with systemic symptoms - DRESS, and acute generalized exanthematous pustulosis - AGEP) accounted for $40.3 \%$ of all reactions. Two-thirds of patients were aged between 21 and 60 years, while the mean age was 47.2 years (range: 3 - 92). More females $(80.0 \%)$ had SJS than males $(20.0 \%)$, but TEN showed a reverse pattern ( $83.3 \%$ males vs $16.7 \%$ females). Overall, the male:female ratio was 1.68:1. Allopurinol was the most common culprit drug causing SJS (7/15) and TEN (2/6). Cotrimoxazole and Cloxacillin were the two most common antimicrobials implicated in CADR, while the most common analgesic was Celecoxib. One-third of our patients took only a single drug, while the average number of drugs taken by a patient was three. Two patients died, one each from dapsone hypersensitivity syndrome and TEN, resulting in a mortality rate of $3.39 \%$.

Conclusion: SJS was the most common CADR encountered in our center, while the most common culprit drug was allopurinol. Antibiotics as a group caused the most CADR.

Keywords: Malaysia; Dermatology; Allopurinol; Anti-Infective Agents

Citation: Ramalingam R. Cutaneous adverse drug reaction: a review of a four-year experience in a tertiary referral hospital in Malaysia. Int J Med Rev. 2017;4(2):43-46. doi: 10.29252/ijmr-040203.

\section{Introduction}

Drug reactions are unwanted reactions of the body following the administration of drugs that are uncharacteristic of the expected pharmacodynamic effects. ${ }^{1}$ These include cutaneous adverse drug reactions (CADR), which range from mild pruritus and transient erythema to severe life-threatening conditions such as Stevens-Johnson syndrome (SJS) and toxic epidermal necrolysis (TEN), with significant morbidity and mortality. Sadly, drug reactions are often underreported, and CADR are often misdiagnosed as viral exanthems or attributed to collagen vascular diseases. This in turn may be due to insufficient awareness by healthcare providers, given the emergence of newer classes of drugs with yet unclassified adverse effects as well as evolving prescription profiles. ${ }^{2,3}$ Hence, it is hoped that this clinical audit and review of literature will create the much needed awareness of the epidemiology of CADR, and subsequently influence future, more judicious prescribing practices of healthcare providers.

\section{Methods}

A retrospective review of all patients referred to the Department of Dermatology of Hospital Tengku Ampuan Afzan (HTAA), Kuantan, Pahang, Malaysia, with CADR from 2013 to 2016 was carried out to determine the epidemiology of CADR in the local population.

Results

A total of 62 CADR involving 59 patients were seen among 7,353 new patients, yielding an incidence rate of $0.80 \%$ (yearly CADR rate range: $0.16-1.89 \%$ ). The highest CADR rate was seen among indigenous peoples (Table 1).

Copyright (C) 2017 The Author(s). This is an open-access article distributed under the terms of the Creative Commons Attribution License (http://creativecommons.org/licenses/by/4.0), which permits unrestricted use, distribution, and reproduction in any medium, provided the original work is properly cited. 
Table 1. Rates of CADR between both genders and various ethnic groups

\begin{tabular}{|c|c|c|c|}
\hline 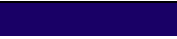 & No. of new patients $\mathbf{N}(\%)$ & No. with CADRN (\%) & CADR rate $(\%)$ \\
\hline \multicolumn{4}{|l|}{ Gender } \\
\hline Male & $3567(48.5)$ & $37(62.7)$ & 1.04 \\
\hline Female & $3786(51.5)$ & $22(37.3)$ & 0.58 \\
\hline \multicolumn{4}{|l|}{ Ethnicity } \\
\hline Malay & 5697 (77.5) & $41(69.5)$ & 0.72 \\
\hline Chinese & $1176(16.0)$ & $11(69.5)$ & 0.94 \\
\hline Indian & $322(4.4)$ & $3(5.1)$ & 0.93 \\
\hline Indigenous & $158(2.1)$ & $4(6.8)$ & 2.53 \\
\hline Total & 7353 & 59 & 0.80 \\
\hline
\end{tabular}

CADR: cutaneous adverse drug reaction

Two-thirds of patients with CADR were between 21 and 60 years old, with a mean age of 47.3 years (range: $3-92$ ). Two patients died, one each from dapsone hypersensitivity syndrome and TEN, resulting in a mortality rate of $3.39 \%$. More females $(80.0 \%)$ had SJS than males (20.0\%), while TEN showed a reverse pattern (83.3\% males vs $16.7 \%$ females). Fig 1 illustrates the various CADR reported in our cohort, while fig 2 highlights the culprit groups of drugs.

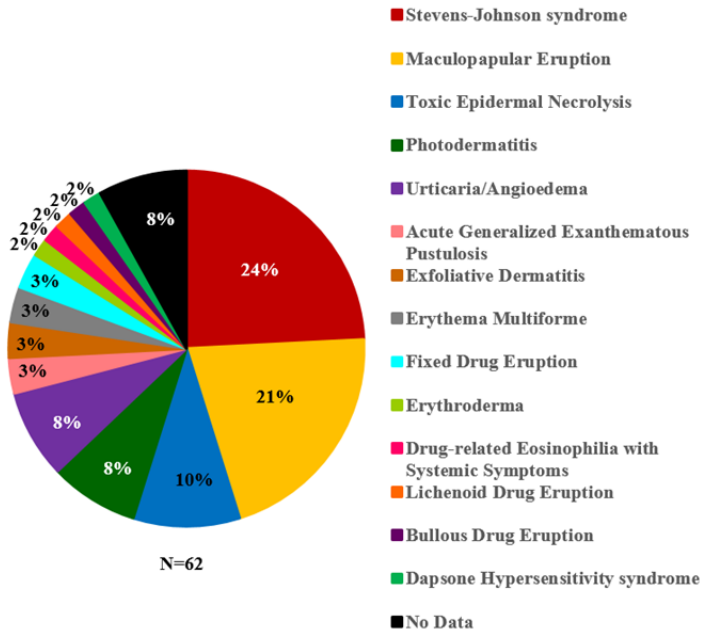

Figure 1. Type of CADR

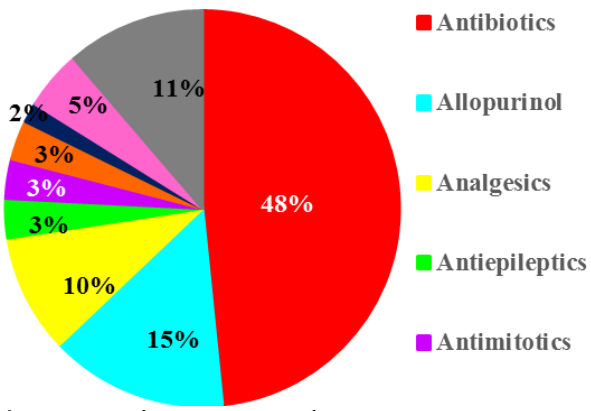

Figure 2. Culprit Groups of Drugs

Allopurinol was the most common culprit drug causing SIS (7/15) and TEN (2/6). Cotrimoxazole and Cloxacillin were the two most common antimicrobials implicated in CADR, while the most common analgesic was Celecoxib. One-third of our patients took only a single drug, while the average number of drugs taken by a patient was three.

\section{Discussion}

Where do we stand against other Asian centers? Table 2 below compares our findings with clinicoepidemiological studies on CADR in Malaysia and various Asian countries. Severe CADR, namely SJS and TEN, were among the most common CADRs observed in the Malaysian hospitals listed above, including ours. This could be due to a referral bias, being dermatology referral centers accepting serious CADRs. This could also explain the lower CADR rate in our cohort compared to $1.38 \%$ and $1.5 \%$ of dermatology referrals in Denmark $^{4}$ and Tunisi ${ }^{5}$, respectively. We now know that there are specific genetic markers for carbamazepineand phenytoin-induced CADRs, namely the HLA$\mathrm{B}^{\star}$ 15:02 allele predicting the risk of carbamazepine ${ }^{6-9}$ as well as phenytoin ${ }^{10-13}$ induced SJS/TEN in South-East Asian populations, HLA-A ${ }^{\star} 31: 01$ allele for carbamazepine-induced hypersensitivity reactions in European populations ${ }^{14}$, and HLA-B ${ }^{\star} 15: 13$ for phenytoin-induced SJS/TEN among Malays in Malaysia $^{10}$, and that these allelic markers occur with varying frequency in different ethnic populations. Likewise, HLA-B ${ }^{\star}$ 13:01 has been reported to be a predictor for dapsone-induced, drug-hypersensitivity syndromes among patients with leprosy. ${ }^{15}$ HLA-B ${ }^{\star}$ 13:01 has also been found among the three main indigenous groups in Peninsular Malaysia, namely the Temuan, Jehai, and Kensiu, with the Kensiu peoples having the highest allele frequency. ${ }^{16}$ Whether this holds true for the indigenous peoples of the state of Pahang, resulting in higher CADR rates among them, requires further pharmacogenomic studies. Antimicrobials being the predominant culprit group in almost all of the studies above not only reflects the high infectious diseases burden in tropical and subtropical Asia, but also serves to remind us of more judicious prescriptions of these agents in the future.

\section{Conclusion}

SJS was the most common CADR encountered in our center, while the most common culprit drug was allopurinol. Antimicrobials as a group caused the most $\mathrm{CADR}$, and indigenous peoples have a high rate of developing CADR. 
Table 2. A comparison of Clinico-Epidemiological Studies on CADR in Malaysia and Various Asian Countries

\begin{tabular}{|c|c|c|c|c|c|c|c|}
\hline & $\begin{array}{l}\text { Huang HY, } \\
\text { et al. } \\
\text { (2004-2008) } \\
\text { Shanghai, } \\
\text { China } \\
\text { N=734 }\end{array}$ & $\begin{array}{l}\text { Choon SE, et } \\
\text { al. }^{18}(2001 \text { - } \\
2010) \text { Johor } \\
\text { Bahru, } \\
\text { MalaysiaN=3 } \\
62\end{array}$ & $\begin{array}{c}\text { Talib NH, et } \\
\text { al. }^{19} \text { (2009- } \\
\text { 2010) Kuala } \\
\text { Lumpur, } \\
\text { MalaysiaN=1 } \\
34\end{array}$ & $\begin{array}{c}\text { Garg HK, et } \\
\text { al. }{ }^{20}(2010- \\
2012) \\
\text { Ajman, UAE } \\
N=43\end{array}$ & $\begin{array}{c}\text { Mokhtari F, } \\
\text { et al. }^{21} \\
\text { (2006-2013) } \\
\text { Isfahan, Iran } \\
\quad \mathrm{N}=282\end{array}$ & $\begin{array}{c}\text { Janardhan B, } \\
\text { et al. } \\
\text { (2013-2014) } \\
\text { Hyderabad, } \\
\text { India } N=481\end{array}$ & $\begin{array}{c}\text { Our Study } \\
(2013-2016) \\
\text { Kuantan, } \\
\text { Malaysia } \\
\text { N=62 }\end{array}$ \\
\hline Male:Female ratio & $1: 1.97$ & $1: 1.14$ & $1: 1.1$ & $1: 1.15$ & $1: 1.55$ & $1: 1.78$ & $1: 1.67$ \\
\hline Mean age (years) & $43.90(8-93)$ & $39.60(1-98)$ & $47(14-91)$ & 30.0 & $\begin{array}{c}29.48(0.4- \\
90)\end{array}$ & $42(1-64)$ & $47.2(3-92)$ \\
\hline $\begin{array}{l}\text { Median latency } \\
\text { (days) }\end{array}$ & $7.64 \pm 8.32$ & NA & NA & $5.63 \pm 0.5$ & NA & $4(1-120)$ & 6 \\
\hline $\begin{array}{l}\text { Incidence/Prevalen } \\
\text { ce }(\%)\end{array}$ & NA & $\begin{array}{c}\text { Incidence: } \\
0.86\end{array}$ & $\begin{array}{c}\text { Prevalence: } \\
0.2\end{array}$ & NA & NA & $\begin{array}{c}\text { Prevalence: } \\
1.08\end{array}$ & $\begin{array}{l}\text { Incidence: } \\
0.8\end{array}$ \\
\hline $\begin{array}{l}\text { Most common } \\
\text { CADR }(\%)\end{array}$ & $\begin{array}{c}1.0 \text { EM } \\
(34.7) 2.0 \\
\text { Urticaria } \\
(26.2) 3.0 \\
\text { MPE (21.7) }\end{array}$ & $\begin{array}{c}\text { MPE (42.3) SJS } \\
(24.3) \text { DRESS } \\
(9.4)\end{array}$ & $\begin{array}{l}\text { MPE (22.4) SJS } \\
(9.7) \text { FDE (8.9) }\end{array}$ & $\begin{array}{c}\text { MPE (48.8) } \\
\text { Erythroderm } \\
\text { a (18.6) } \\
\text { Urticaria } \\
(11.7)\end{array}$ & $\begin{array}{l}\text { SJS (31.9) } \\
\text { MPE }(24.5) \\
\text { TEN (11.0) }\end{array}$ & $\begin{array}{c}\text { MPE (35.6) } \\
\text { Urticaria } \\
(26.2) \text { FDE } \\
(17.9)\end{array}$ & $\begin{array}{l}\text { SJS }(24.2) \\
\text { MPE }(21.0) \\
\text { TEN }(9.7)\end{array}$ \\
\hline $\begin{array}{l}\text { Most common } \\
\text { groups of culprit } \\
\text { drugs }\end{array}$ & $\begin{array}{c}1.0 \\
\text { Antimicrobia } \\
\text { Is }(48.3) 2.0 \\
\text { Allopurinol } \\
(6.0)\end{array}$ & $\begin{array}{c}1.0 \\
\text { Antimicrobials } \\
(40.3) 2.0 \\
\text { AEDs }(22.4) \\
3.0 \\
\text { Allopurinol } \\
(13.8)\end{array}$ & $\begin{array}{c}1.0 \\
\text { Antimicrobials } \\
(36.6) 2.0 \\
\text { TCM (17.9) } \\
\text { 3.0 Analgesics } \\
(13.4)\end{array}$ & $\begin{array}{c}1.0 \\
\text { Antimicrobia } \\
\text { Is (48.8) } 2.0 \\
\text { Analgesics } \\
(32.5) 3.0 \\
\text { TCM (4.6) }\end{array}$ & $\begin{array}{c}\text { 1.0 AEDs } \\
(51.8) 2.0 \\
\text { Antimicrobia } \\
\text { Is (33.7) } 3.0 \\
\text { Analgesics } \\
(5.7)\end{array}$ & $\begin{array}{c}1.0 \\
\text { Antimicrobia } \\
\text { Is (56.3) } 2.0 \\
\text { NSAIDs } \\
(19.5) 3.0 \\
\text { AEDs }(16.6)\end{array}$ & $\begin{array}{c}1.0 \\
\text { Antimicrobia } \\
\text { Is (48.4) } 2.0 \\
\text { Allopurinol } \\
(14.5) 3.0 \\
\text { Analgesics } \\
(9.7)\end{array}$ \\
\hline
\end{tabular}

EM: Erythema Multiforme; MPE: Maculopapular Exanthem; SJS: Stevens-Johnson Syndrome; DRESS: Drug-Related Eosinophilia with Systemic Symptoms; FDE: Fixed Drug Eruption; TEN: Toxic Epidermal Necrolysis; AEDs: Anti-Epileptic Drugs; TCM: Traditional and Complementary Medicine; NSAIDs: Non-Steroidal Anti-Inflammatory Drugs; NA: Not Available

\section{Ethical Approval}

Not applicable.

\section{References}

1. Roujeau J-C, Allanore L, Liss Y, Mockenhaupt M. Severe cutaneous adverse reactions to drugs (SCAR): definitions, diagnostic criteria, genetic predisposition. Dermatol Sinica. 2009;27(2):203-9.

2. Lazarou J, Pomeranz BH, Corey PN. Incidence of adverse drug reactions in hospitalized patients: a meta-analysis of prospective studies. JAMA. 1998;279(15):1200-5. pmid: 9555760.

3. Coopman SA, Johnson RA, Platt R, Stern RS. Cutaneous disease and drug reactions in HIV infection. N Engl J Med. 1993;328(23):1670-4. doi: 10.1056/NEJM199306103282 304. pmid: 8487826 .

4. Borch JE, Andersen KE, Bindslev-Jensen C. Cutaneous Adverse Drug Reactions Seen at a University Hospital Department of Dermatology. Acta Dermato-Venereol. 2006;86(6):523-7. doi: 10.2340/00015555-0153.

5. Zaraa I, Jones M, Trojjet S, Cheikh Rouhou R, El Euch D, Mokni $M$, et al. Severe adverse cutaneous drug eruptions: epidemiological and clinical features. Int J Dermatol. 2011;50(7):877-80. doi: 10.1111/j.1365-4632.2010.04785 .x. pmid: 21699528.

6. Lim KS, Kwan P, Tan CT. Association of HLA-B* 1502 allele and carbamazepine-induced severe adverse cutaneous drug reaction among Asians, a review. Neurol Asia. 2008;13(6):15-21.

7. Chang CC, Too CL, Murad S, Hussein SH. Association of

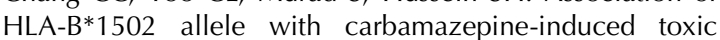
epidermal necrolysis and Stevens-Johnson syndrome in the multi-ethnic Malaysian population. Int J Dermatol. 2011;50(2):221-4. doi: 10.1111/j.1365-4632.2010.04745 .x. pmid: 21244392.

8. Kulkantrakorn K, Tassaneeyakul W, Tiamkao S, Jantararoungtong T, Prabmechai N, Vannaprasaht S, et al.

\section{Conflicts of Interests Disclosure}

None.

\section{Funding}

None.

HLA-B*1502 strongly predicts carbamazepine-induced Stevens-Johnson syndrome and toxic epidermal necrolysis in Thai patients with neuropathic pain. Pain Pract. 2012;12(3):202-8. doi: 10.1111/j.1533-2500.2011.00479 .x. pmid: 21676164.

9. Wang Q, Zhou JQ, Zhou LM, Chen ZY, Fang ZY, Chen SD, et al. Association between HLA-B*1502 allele and carbamazepine-induced severe cutaneous adverse reactions in Han people of southern China mainland. Seizure. 2011;20(6):446-8. doi: 10.1016/j.seizure.2011.02.003. pmid: 21397523.

10. Chang CC, Ng CC, Too CL, Choon SE, Lee CK, Chung WH, et al. Association of HLA-B*15:13 and HLA-B*15:02 with phenytoin-induced severe cutaneous adverse reactions in a Malay population. Pharmacogenomics J. 2017;17(2):170-3. doi: 10.1038/tpj.2016.10. pmid: 26927288.

11. Cheung YK, Cheng SH, Chan EJ, Lo SV, Ng MH, Kwan P. HLA-B alleles associated with severe cutaneous reactions to antiepileptic drugs in Han Chinese. Epilepsia. 2013;54(7):1307-14. doi: 10.1111/epi.12217. pmid: 23692 434.

12. Locharernkul C, Loplumlert J, Limotai C, Korkij W, Desudchit T, Tongkobpetch $\mathrm{S}$, et al. Carbamazepine and phenytoin induced Stevens-Johnson syndrome is associated with HLA-B*1502 allele in Thai population. Epilepsia. 2008;49(12):2087-91. doi: 10.1111/j.1528-1167.2008.01 719.x. pmid: 18637831.

13. Hung SI, Chung WH, Liu ZS, Chen $\mathrm{CH}$, Hsih MS, Hui RC, et al. Common risk allele in aromatic antiepileptic-drug induced Stevens-Johnson syndrome and toxic epidermal necrolysis in Han Chinese. Pharmacogenomics. 2010;11(3):349-56. doi: 10.2217/pgs.09.162. pmid: 20235 791. 
14. McCormack $M$, Alfirevic A, Bourgeois $S$, Farrell II, Kasperaviciute D, Carrington M, et al. HLA-A*3101 and carbamazepine-induced hypersensitivity reactions in Europeans. N Engl J Med. 2011;364(12):1134-43. doi: 10.1056/NEJMoa1013297. pmid: 21428769.

15. Wang N, Parimi L, Liu H, Zhang F. A Review on Dapsone Hypersensitivity Syndrome Among Chinese Patients with an Emphasis on Preventing Adverse Drug Reactions with Genetic Testing. Am J Trop Med Hyg. 2017;96(5):1014-8. doi: 10.4269/ajtmh.16-0628. pmid: 28167593.

16. Jinam TA, Saitou N, Edo J, Mahmood A, Phipps ME. Molecular analysis of HLA Class I and Class II genes in four indigenous Malaysian populations. Tissue Antigens. 2010;75(2):151-8. doi: 10.1111/j.1399-0039.2009.01417 .x. pmid: 20003135

17. Huang $\mathrm{HY}$, Luo XQ, Chan LS, Cao ZH, Sun XF, Xu JH. Cutaneous adverse drug reactions in a hospital-based Chinese population. Clin Exp Dermatol. 2011;36(2):135-41. doi: 10.1111/j.1365-2230.2010.03922.x. pmid: 20738322.

18. Choon SE, Lai NM. An epidemiological and clinical analysis of cutaneous adverse drug reactions seen in a tertiary hospital in Johor, Malaysia. Indian J Dermatol Venereol Leprol. 2012;78(6):734-9. doi: 10.4103/0378-6323.102367. pmid: 23075643.

19. Talib $\mathrm{NH}$, Leelavathi $\mathrm{M}$, Hamzah Z. Common adverse cutaneous drug reaction patterns and the causative drugs in Malaysia. South Afr Fam Pract. 2015;57(4):227-30. doi: 10.1080/20786190.2015.1024026.

20. Garg HK, John LJ, Thomas IN, Muttappallymyalil Jk, Kadhum W, Sreedharan J. Variety and Incidence of Cutaneous Adverse Drug Reactions in a UAE Hospital. Int J Med Res Prof. 2016;2(5). doi: 10.21276/ijmrp.2016.2.5.009.

21. Mokhtari F, Nikyar Z, Naeini BA, Esfahani AA, Rahmani $S$ Adverse cutaneous drug reactions: Eight year assessment in hospitalized patients. J Res Med Sci. 2014;19(8):720-5 pmid: 25422656.

22. Janardhan B, Shailendra D. Prevalence and pattern of adverse cutaneous drug reactions presenting to a tertiary care hospital. Int J Res Dermatol. 2017;3(1):74. doi: 10.18203/issn.2455-4529.IntJResDermatol20164248. 